\title{
Protein and RNA quantification of multiple genes in single cells
}

\author{
Ibrahim Kays \& Brian Edwin Chen*,1,2
}

\section{ABSTRACT}

Single-cell analysis overcomes the problems of cellular heterogeneity by revealing the individual differences between cells in tissue. The current tools used to profile gene expression at the single-cell level are arduous and often require specialized equipment. We have previously developed a technique to quantify protein expression levels in single living cells. Here, we combine quantification of protein expression with absolute measurement of mRNA amounts of the same gene in the same cell, to profile the expression of genes at the transcriptional and translational levels. We show that high heterogeneity exists at both the mRNA and protein levels for multiple genes, even among monoclonal cells. We demonstrate a rapid, straightforward approach to single-cell profiling of RNA and protein production.

\section{METHOD SUMMARY}

We report a fast and reliable technique for the quantification of endogenous protein and mRNA levels of any gene from single cells.

KEYWORDS

CRISPR-Cas $9 \cdot$ fluorescence imaging - genome editing $\cdot$ mRNA quantification $\cdot$ protein quantification $\cdot$ singlecell analysis

Centre for Research in Neuroscience, Research Institute of the McGill University Health Centre, Montréal, Québec, H3G 1A4, Canada, ${ }^{2}$ Departments of Medicine and Neurology \& Neurosurgery, McGill University, Montréal, Québec, H3G 1A4, Canada

*Author for correspondence: brian.chen@ mcgill.ca

BioTechniques 66: 15-21 (January 2019)

10.2144/btn-2018-0130

First draft submitted: 4 September 2018; Accepted for publication: 27 September 2018

\section{INTRODUCTION}

Understanding the relationship between genes and phenotypes is a central component of biology. Profiling the expression of genes with respect to mRNA or protein products has advanced our fundamental understanding of cell biology, such as the maintenance of cell structure, progression of cell cycle and neural development, as well as how abnormalities in gene expression can lead to disease states. Several analytic methods have been developed to profile gene expression at the level of mRNA or protein. For example, microarrays and RNA sequencing approaches can identify mRNA from tens of thousands of different genes whereas protein expression can be assayed using spectrometry, chromatography, immunoassays and gel electrophoresis $[1,2]$.

The current tools to assay mRNA and protein expression are generally performed on large pools of cells or tissues. Large numbers of cells can increase the reliability of results and overcome issues of low sensitivity or the low signal-to-noise ratio of common assays. However, it is becoming increasingly clear that even neighboring cells within tissues or pools of clonal cells are not homogenous [3-8]. Cellular dynamics, such as differing transcriptional and translational states, cell signaling, cell cycle, development, and other molecular processes in addition to stochasticity together produce cellular heterogeneity, even in immortalized clonal cell lines. Assays performed on large numbers of cells result in population-averaged measurements that miss important individual cellular differences, and the results obtained from population samples cannot be translated into the single-cell scale. Therefore, analysis of single cells is required to identify hidden molecular mechanisms that are masked by the population average.

We have previously developed a technique that allows for quantitation of protein levels in single cells in vivo [5]. This protein quantitation ratioing $(\mathrm{PQR})$ technique uses a genetic tag that produces a stoichiometric ratio of a fluorescent protein reporter and the protein of interest during protein translation. The fluorescence intensity is proportional to the number of molecules of the protein of interest produced and is used to determine the relative protein amount within the cell (Figure 1A). Using genome editing tools, $\mathrm{PQR}$ constructs can be inserted into any endogenous genomic locus to quantify endogenous protein levels in single living cells [5]. Cells expressing PQR constructs can be noninvasively imaged over time using standard fluorescence microscopy, allowing for other biological assays matched at the single-cell level, such as sequencing, mRNA expression analysis and pharmacological manipulations. For example, we used single-cell protein and mRNA quantification in single cells from human and mouse to determine the covariation between mRNA expression and protein amounts. We found that mRNA expression is a weak predictor of protein translation [5]. Recently, simultaneous measurement of RNA and protein was also performed in single cells using microfluidics, and a high heterogeneity and poor mRNA-to-protein correlations were also observed [9-11]. Current RNA analysis techniques such as RNA-Seq and microarrays, while amenable to single cells, are still costly and require deep coverage and sufficiently long reads of sequenced areas in order to accurately produce the sequence; particularly for transcripts expressed at low abundance [12].

Therefore, despite recent efforts to profile global gene expression from single cells, straightforward and inexpensive protocols are still not available. Here, we describe methods and protocols for fast and accessible quantification of gene expression by measuring both its absolute mRNA transcript numbers and level of protein translation in the same cell (Figure 1B). This tool can be used by any researcher with access to a standard 


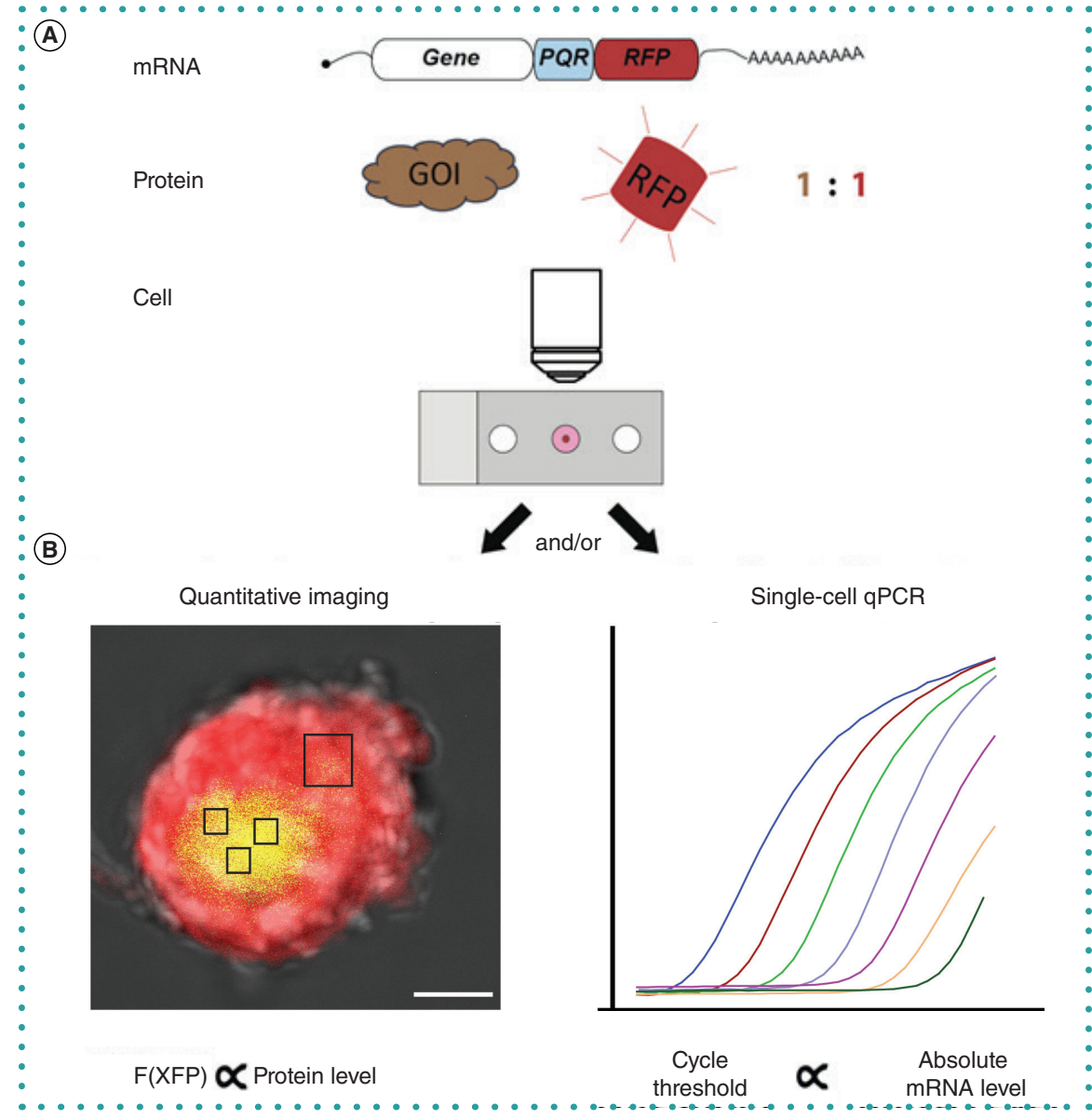

Figure 1. Workflow of protein and mRNA measurement from the same cell. (A) Insertion of a PQR reporter between a fluorescent reporter $(R F P)$ and a $\mathrm{GOI}$ results in the stoichiometric cotranslation of a fluorescent reporter that can be used to quantify endogenous protein production levels. $P Q R$ constructs allow for one molecule of RFP to be produced for each molecule of protein of interest translated. Since the fluorescence output of GFP is directly proportional to its concentration, the fluorescence intensity of the cell can be used to determine the level of production of the protein of interest. (B) Quantitative fluorescence intensity measurement from regions of interest within the cell can be used to quantitate the production level of the upstream protein. Following imaging, the same cell is lysed for total RNA extraction and absolute mRNA abundance quantification using single-cell qPCR. Black boxes are representative regions of interest used for pixel intensity quantification.

GOI: Gene of interest; PQR: Protein quantitation ratioing.

- fluorescence microscope and real-time PCR instrument and can be easily applied to examine single cells obtained from sources such as dissociated tissue samples, FACS sorting or patient-derived stem cells. In addition, these methods are amenable to any cell type such as neurons, immune cells, immortalized cell lines in adherent or suspension form, and dissociated animal cells. Multiple genes with vastly different expression properties can be assayed simultaneously from the same cell, allowing the examination of gene co-regulation at both the transcriptional and translational levels.

Compared with existing single-cell protein or single-cell mRNA assays, our protocol requires $2.5 \mathrm{~h}$ from start to finish, is relatively inexpensive and can be performed using common laboratory tools.

\section{MATERIALS \& METHODS}

Detailed reagents, protocols, experimental setup and troubleshooting information are presented in the supplementary information file.

\section{Experimental design}

We describe a method to first measure the level of protein abundance using a PQR fluorescent reporter, and then quantify the absolute mRNA copy number of that gene in a single cell (Figure 1B). As a source of single cells, we chose the antibodyproducing monoclonal hybridoma mouse cell line $22 \mathrm{c} 10$ because they are large, easy to manipulate, and constantly secrete high levels of antibody containing kappa-isotype immunoglobulin light chains [13]. The insertion of a $P Q R-R F P$ reporter after the constant exon of the kappa light chain gene ensures the production of one molecule of RFP with each kappa light chain protein molecule translated (i.e., an antibody production reporter) (Figure 2A).

By contrast, we also quantified the RPL13a gene, which encodes for ribosomal protein L13a. RPL13a is expressed in all eukaryotic cells at consistent levels, and is routinely used as a reference 'housekeeping' gene for normalization of quantitative mRNA and protein measurements [14-16]. The quantification of RPL13a by the insertion of a PQR-GFPnols reporter at the end of its coding sequence (Figure 2A) can therefore be used as a measure of a cell's individual transcriptional and translational status [5].

Here, we take advantage of the differences between IgK and RPL13a expression to highlight how this technique can be used to profile the transcriptional and translational landscape of multiple genes simultaneously at single-cell resolution (Figure 2B). To generate $22 \mathrm{c} 10$ cells carrying $P Q R$ reporters at both the endogenous RPL13a and $I g K$ loci, we first established a genomeedited cell line with a PQR-GFPnols at the RPL13a locus, and then targeted the $\lg K$ locus with a $P Q R-R F P$ reporter. The nucleolar (nols) targeting sequence on GFP in RPL13a-edited cells sequesters it into the nucleolus, a cellular organelle 100-times smaller than the cytoplasm in volume [17]. Sequestering fluorescent proteins into small cellular organelles increases their local concentration to produce brighter signals that facilitate fluorescence quantification and cell manipulation [5]. The colors of the fluorescent proteins can also be swapped, for example starting with an IgK-PQR-GFP edited cell line first (Supplementary Figure 1B).

We also apply this method on cells dissociated from genome-edited Drosophila that have a different color PQR reporter inserted into each parental allele to examine the relationship between paternal and maternal mRNA and protein expression in each cell. 
Detailed information and protocols on the generation of PQR-edited single cells, quantitative imaging, mRNA reverse transcription, qPCR and data analysis are presented in the Supplementary file.

\section{Cell culture}

Genome-edited 22c10 cells were cultured at $37^{\circ} \mathrm{C}$ under $5 \% \mathrm{CO}_{2}$ in $\mathrm{H}$-Cell (22c10) or DMEM (HEK293T), supplemented with $100 \mathrm{U} / \mathrm{ml}$ penicillin and $100 \mu \mathrm{g} / \mathrm{ml}$ streptomycin. For genome editing experiments, $800 \mathrm{ng}$ of CRISPR-Cas9 plasmid DNA were co-transfected with $800 \mathrm{ng}$ of repair template circular plasmid in 12-well plates. After 2-7 days, fluorescent cells were manually isolated into single cell clones and expanded before verification of correct genome editing (see [5] for detailed methods). Double knockin cell lines were generated by first inserting a $P Q R$ GFPnols reporter construct into the endogenous $R P L 13 a$ locus to make a stable green fluorescent cell line. A PQR-RFP reporter construct was subsequently inserted into the kappa light chain immunoglobulin locus and cells that were fluorescent in both the green and red channels were selected for single-cell clonal expansion.

Protein quantitation reporter constructs Protein quantitation reporter constructs were designed and constructed as described previously [5] and are available from Addgene.

\section{Image acquisition \& analysis}

Fluorescence and brightfield microscopy was performed using a Zeiss AxioScope A1. All images were acquired at $1388 \times 1040$ pixels using a $40 \times$ water objective, N.A. 1.0 (epifluorescence) (Supplementary figure 2D). Fluorescence emission was detected using a charge-coupled device (CCD) camera (MRm). All image acquisition parameters were fixed for each imaging channel for exposure time, excitation intensity and gain. Cells that were dimmer or brighter than the fixed initial acquisition dynamic range were not included for analysis.

Images were selected for analysis based on identification of single healthy cells and low background. Fluorescence pixel intensities were measured in several random regions of interest (ROIs) within the cell cytoplasm (IgK) or nucleolus (RPL13a) using Image J. Average pixel intensities were

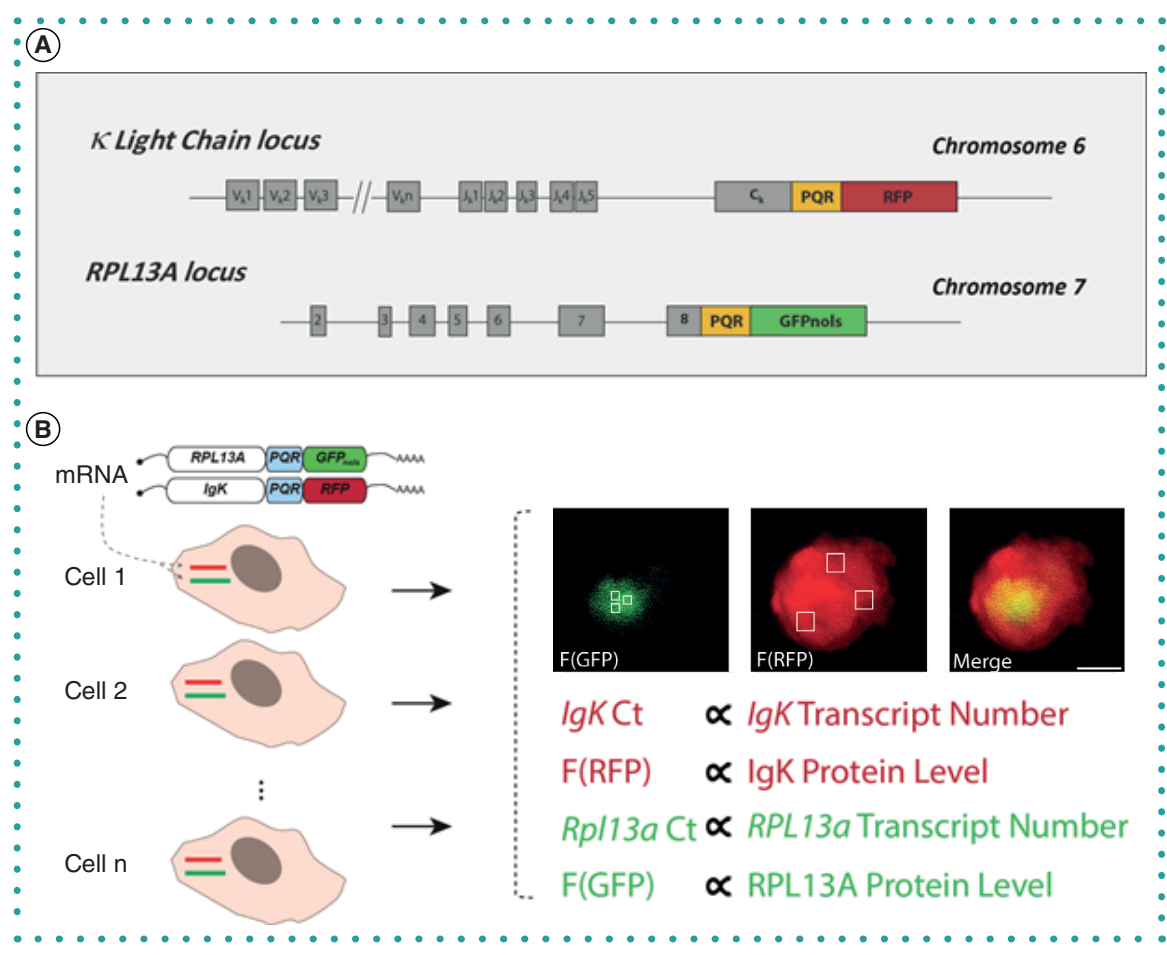

Figure 2. Protein and mRNA measurement for multiple genes in a single cell. (A) The fluorescent reporters (approximately $800 \mathrm{bp}$ in size) are inserted in-frame immediately upstream of the stop codon, preserving the endogenous $5^{\prime}$ and 3 ' untranslated regions and the native coding sequence. PQR constructs carrying an RFP and a GFPnols reporter were inserted into the endogenous loci of $R P L 13 a$ and $I g K$, respectively, in 22c10 cells. (B) The transcript numbers and protein levels of both genes from the same cell can be obtained using single-cell qPCR and quantitative imaging. Representative example of a double knock-in 22c10 cell expressing PQR-GFPnols from the endogenous $R P L 13 a$ locus and $P Q R-R F P$ from the endogenous $I g K$ locus. White boxes are representative regions of interest used for pixel intensity quantification. Boxes placed in the nucleolus were used to quantify GFP (RPL13a) levels, and boxes placed in the cytoplasm were used to determine RFP (IgK) levels. $P Q R$ : Protein quantitation ratioing.

calculated from five ROIs of $10 \times 10$ pixels for measurements within the cytoplasm and $5 \times 5$ pixels for measurements within nucleoli (Figure 2B). All signal intensities were background subtracted from the average of three $10 \times 10$ ROIs surrounding the cell.

\section{Real-time quantitative PCR}

Detailed information and protocols describing single-cell isolation, RNA extraction, reverse-transcription and qPCR are presented as supplementary information. Briefly, total RNA was extracted from single cells using a modified Trizolbased protocol, and resuspended in $6 \mu \mathrm{l}$ of $\mathrm{ddH}_{2} \mathrm{O}$. Reverse-transcription reactions were performed with gene-specific cocktails $(0.1 \mu \mathrm{M}$ final concentration of each primer) using Superscript IV reverse polymerase (Thermo Fisher, MA, USA). $6 \mu$ l of cDNA was used as input template for real-time PCR using the TaqMan Fast Advanced Mastermix (Thermo Fisher).
Real-time amplification was measured using the StepOnePlus real-time PCR system (Thermo Fisher) and cycle threshold values were calculated using StepOne software (Thermo Fisher). Absolute quantification of RPL13a and IgK mRNA copy numbers was determined using standard curves generated with synthesized oligonucleotide standards containing the RPL13a-PQR-XFP and IgK$P Q R-X F P$ target. Primers and doublequenched FAM/ZEN/lowaBlackFQ probes were purchased from Integrated DNA Technologies (IA, USA) (all primer and probe sequences are shown in Table 1). See supplementary information for replicates and controls.

\section{Statistical analysis}

Linear correlations were calculated by fitting the data to a simple linear regression model, with the coefficient of determination, $R^{2}$. We tested the null 
TABLE 1. Sequences of primers and probes used.

\begin{tabular}{|l|l|l|l|l|}
\hline & Forward primer & Reverse primer & RT primer & qPCR probe \\
\hline Mouse $R P L 13 a$ & TCCCTCCACCCTATGACAAG & $\begin{array}{l}\text { GTCACTGCCTGG- } \\
\text { TACTTCC }\end{array}$ & $\begin{array}{l}\text { GCAGCCCTGCTACT- } \\
\text { CATTTTC }\end{array}$ & $\begin{array}{l}\text { GATGTAGAA- } \\
\text { GAAAACCCTGGACC }\end{array}$ \\
\hline Human $R P L 13 a$ & TGTTTGACGGCATCCCAC & $\begin{array}{l}\text { CTGTCACTGCCTGG- } \\
\text { TACTTC }\end{array}$ & $\begin{array}{l}\text { CTGCTGGCCACATTT- } \\
\text { TATGTC }\end{array}$ & $\begin{array}{l}\text { CTTCAGACGCAC- } \\
\text { GACCTTGAGGG }\end{array}$ \\
\hline IgK & AGTGGAAGATTGATGGCAGTG & $\begin{array}{l}\text { CTGTCTTTGCTGTCCT- } \\
\text { GATCA }\end{array}$ & $\begin{array}{l}\text { GGTG- } \\
\text { GATTTCAGGGCAACTA }\end{array}$ & $\begin{array}{l}\text { ACAAAATGGCGTCCT- } \\
\text { GAACAGTTGG }\end{array}$ \\
\hline $\begin{array}{l}\text { Mouse } R P L 13 a \text { outside } \\
\text { of homology arms }\end{array}$ & CGGGTTGCTAACCTGGAATA & $\begin{array}{l}\text { CAGTCTCCAT- } \\
\text { CAAGGGGAAA }\end{array}$ & & \\
\hline $\begin{array}{l}\text { lgK outside of homology } \\
\text { arms }\end{array}$ & & $\begin{array}{l}\text { TAACTGGGGGAAGGG- } \\
\text { CACT }\end{array}$ & & \\
\hline
\end{tabular}

hypothesis that the variables were independent of each other and that the true $R^{2}$ value was 0 .

\section{RESULTS \& DISCUSSION}

The PQR fluorescent reporters are translated stoichiometrically each time RPL13a or IgK protein molecules are produced. The fluorescence intensity of the cell (in arbitrary units) is proportional to the concentration of fluorescent reporter, which correlates directly with the level of translation of the protein of interest. Therefore, the fluorescence intensity or brightness of the cell can be used as a measure of the amount of production or steady state level of the protein of interest [5]. Similarly, the cycle threshold of the real-time amplification reaction is proportional to the concentration of input nucleic acid, and this is used to quantify the

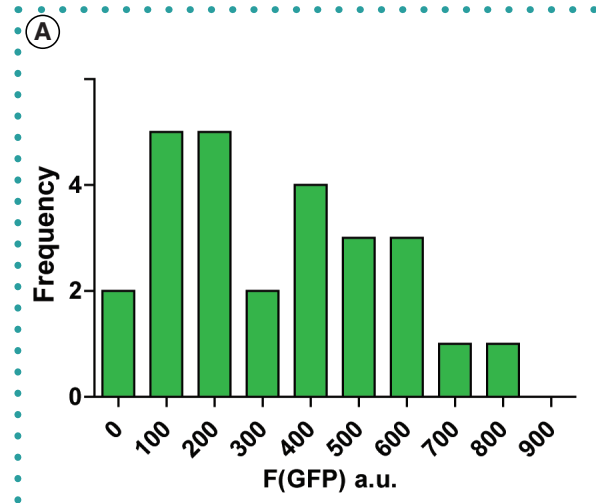

(C)

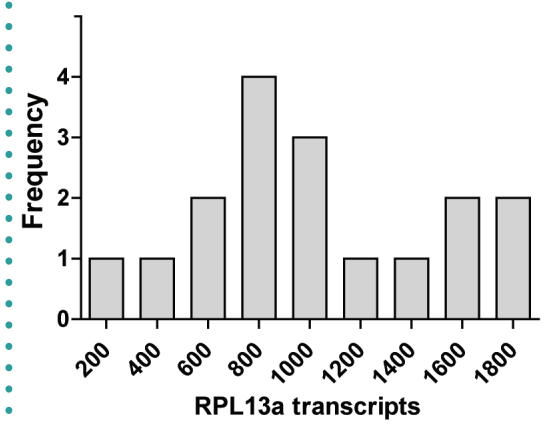

(B)

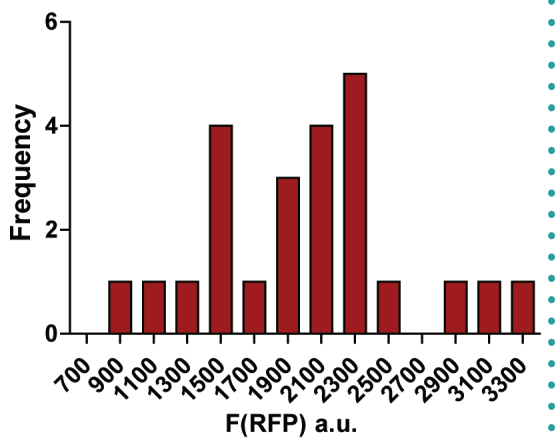

(D)

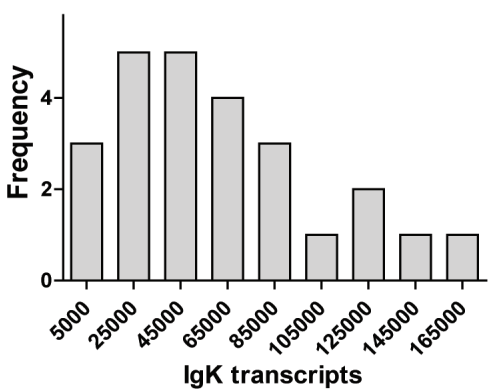

relative or absolute levels of the target mRNA [18]. Measuring the fluorescence intensity of the cell and $\mathrm{Ct}$ values from the qPCR amplification can therefore be used to assay the gene expression of any gene(s) from the same cell, and its variation between cells at both the transcriptional and translational levels.

To determine the levels of $/ g K$ and RPL13a protein in single 22c10 cells, we used CRISPR-Cas9 to generate a cell line carrying a $P Q R-R F P$ and PQR-GFPnols insertion at the endogenous $I g K$ and RPL13a genomic loci, respectively. Single cells with both red and green fluorescence were isolated to derive single-cell clones. The level of expression of the PQR fluorescent reporter correlates with the expression of the target gene and therefore the relative levels of $I g K$ and $R P L 13$ a protein were determined by quantifying the nucleolar GFP fluorescence and cytoplasmic RFP fluorescence intensities from a single cell, respectively. The same cell was then directly lysed and the absolute abundance of $\lg K$ and RPL13a mRNA transcripts were determined using quantitative real-time PCR (Figure 3).

From quantitative imaging of single 22c10 cells, we found that RPL13a protein levels, as measured by nucleolar GFP fluorescence intensity, ranged from 91 to 842 arbitrary units (a.u.) with an average of $364 \pm 205$ (mean $\pm S D ; n=26$ ). By contrast, cytoplasmic RFP fluorescence intensities ranged from 936 to 3263 a.u, with an average of $2004 \pm 619$ a.u $(n=24)$ (Figure 3B).

Using a standard curve of serially diluted known amounts of IgK and RPL13a synthetic oligonucleotide DNA templates, the absolute number of $I g K$ and $R P L 13 a$ mRNA molecules was determined (Figure 3C \& D). We found that the number of RPL13a mRNA transcripts
Figure 3. Endogenous RNA and protein quantification from single cells. (A) GFP fluorescence intensity from single genome-edited $22 \mathrm{c} 10$ cells shows a moderate distribution; $\mathrm{n}=26$. (B) RFP fluorescence intensities were higher and clustered more, indicating consistently high level IgK production; $\mathbf{n}=24$. (C) Frequency distributions of absolute RPL13a mRNA numbers shows moderate expression of the RPL13a gene; $\mathrm{n}=17$. (D) Absolute IgK mRNA levels were relatively higher, indicating high expression of the $I g K$ gene compared to $R P L 13 a ; n=24$. a.u.: Arbitrary units; GFP: Green fluorescent protein; RFP: Red fluorescent protein. 
in single 22c10 cells ranged from 287 to 3023 transcripts, with an average of 1156 mRNA transcripts (Figure 3C). Similarly, IgK mRNA amounts ranged from 2420 to 159,000 transcripts, with an average of $63,835 \mathrm{lgK}$ mRNA transcripts per cell (Figure 3D) (See Supplementary Figure 3B and Supplementary Table 1). This is to be expected as $22 \mathrm{c} 10$ cells are designed and selected to express high levels of kappa light chain $[19,20]$, while RPL13a is a non-essential housekeeping gene [21].

To determine the relationship between gene expression at the transcriptional and translational levels, we analyzed the correlation between the mRNA and protein levels of $I g K$ and $R P L 13 a$ and found interesting differences that shed light on the variety of mRNA-to-protein relationships that exists for different genes, and the importance of examining gene expression at both the mRNA and protein level. For example, RPL13a mRNA levels were not correlated with RPL13a protein levels, with a correlation coefficient of $0.1(p>0.005 ; n=26)$ (Figures 4A \& E). In sharp contrast, IgK mRNA levels were a better predictor of $I g K$ protein levels, with a correlation coefficient of 0.44 $(p<0.005 ; n=24)$ (Figures 4B \& E), which is similar to the global correlation between mRNA and protein abundances [22-24].

To determine whether levels of RPL13a protein co-varied with $I g K$ protein, the red and green fluorescence intensities in single 22 c10 cells were compared. We found no significant linear correlation $\left(R^{2}=0.14\right.$; $\mathrm{p}>0.05 ; \mathrm{n}=24$ ) (Figure 4C). By contrast, to determine whether the expression of IgK and RPL13a mRNA might be linked, we compared absolute IgK and RPL13a transcript numbers. We found that RPL13a transcript numbers were a better predictor of $\lg K \mathrm{mRNA}$ levels $\left(\mathrm{R}^{2}=0.3 ; \mathrm{p}<0.05 ; \mathrm{n}=17\right)$, compared with protein levels (Figure 4D). Time lapse imaging of RPL13a and I $g$ K protein synthesis dynamics would reveal if there is a time-varying correlation between the two, given that RPL13a is itself involved in protein synthesis.

To determine how RPL13a mRNA and protein levels are correlated in human cell lines, we generated human embryonic kidney 293 (HEK293) cells carrying a PQRGFP insertion at the endogenous human $R P L 13 a$ locus. Using the same approach, we found that $\mathrm{h} R P L 13$ a protein levels ranged

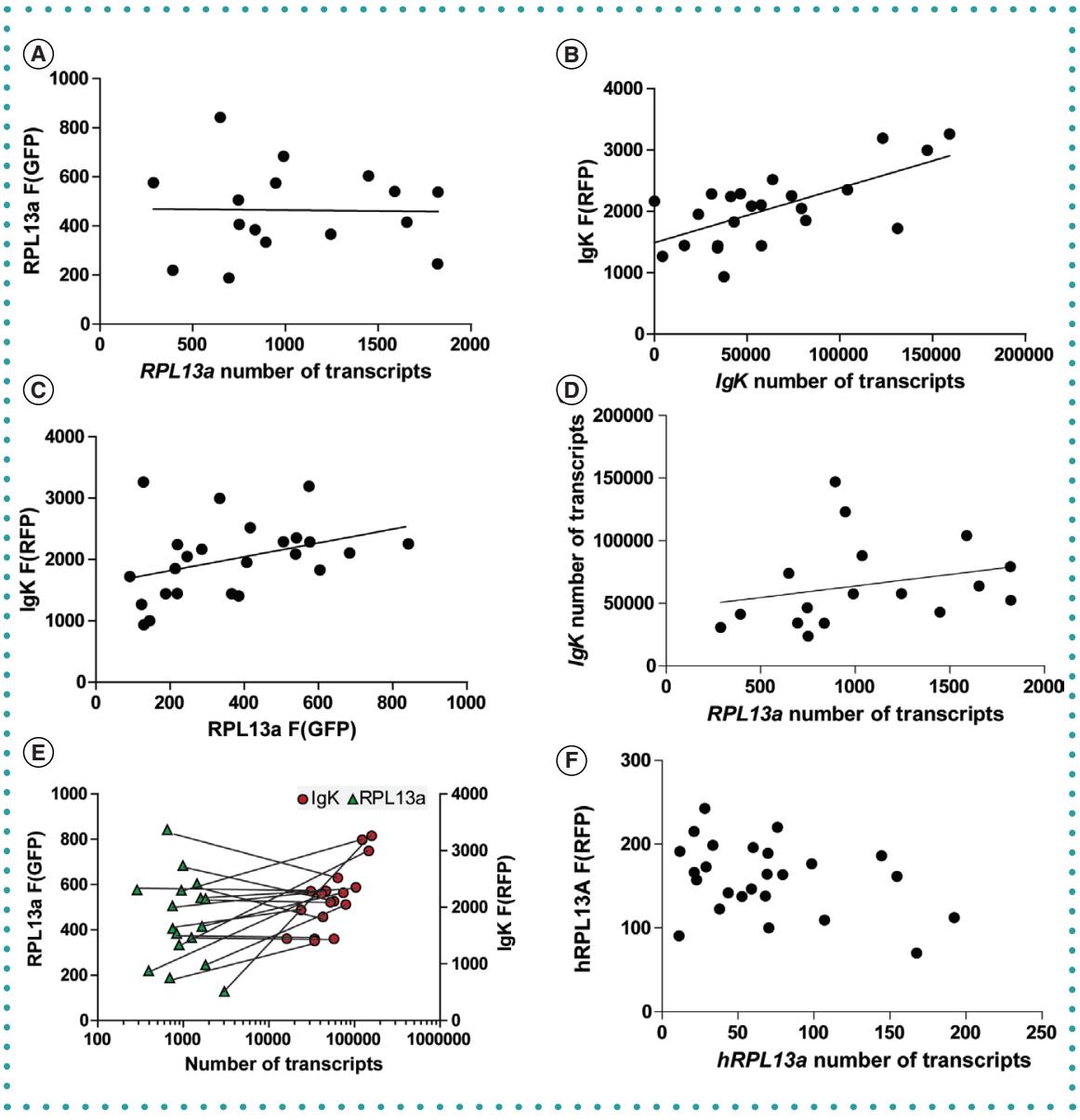

Figure 4. Protein and mRNA relationships between multiple genes in single cells. (A) RPL13a mRNA levels did not correlate with green fluorescence intensities indicating RPL13a mRNA is a weak predictor of $R P L 13 a$ protein production $\left(\mathrm{R}^{2}=0.0 ; \mathrm{p}>0.05 ; \mathrm{n}=17\right)$. (B) $\mathrm{IgK} \mathrm{mRNA}$ levels had a moderate correlation with red fluorescence intensities $\left(R^{2}=0.44 ; p<0.05 ; n=24\right)$. (C \& D) IgK and $R P L 13 a$ exhibit nonsignificant correlations at both the protein level $\left(R^{2}=0.14 ; p>0.05 ; n=24\right)$, and at the mRNA level $\left(R^{2}=0.06\right.$; $p>0.05 ; n=17$ ). (E) Examination of mRNA and protein levels of multiple genes simultaneously from the same cell allows the co-correlation of $m R N A$ and protein levels. Black lines connect data obtained from the same cell $(\mathrm{n}=17)$. ( $\mathrm{F})$ Human RPL13a $(\mathrm{h} R P L 13 a)$ mRNA levels were also a poor predictor of red fluorescent intensities $\left(R^{2}=0.12 ; p>0.05 ; n=25\right)$, indicating a similar kind of relationship between RPL13a mRNA and protein across the mouse and human genomes.

from 60 to 240 a.u. (compared with 91 to 842 a.u, for mouse RPL13a), and absolute hRPL13a mRNA transcript numbers ranged from 11 to 192 (compared with 287 to 3023 mouse RPL13a transcripts), resulting in a similarly poor inverse correlation between hRPL13a mRNA and protein levels $\left(\mathrm{R}^{2}=0.12\right.$; $\mathrm{p}>0.05 ; \mathrm{n}=25$ ) (Figure 4F). These results demonstrate similar mRNA-protein relationships for RPL13a between the human and mouse genomes.

Using the genome-edited transheterozygous $R P L 13 a-P Q R-$ RFPnols/RPL13a-PQR-BFPnols flies, we can track both the paternal and maternal RPL13a allele expression using RFPnols and BFPnols RNA and protein, respectively
(Figure 5A). Using the approach described above, we determined that single epidermal cells dissociated from whole flies contained maternal RPL13a transcript numbers that ranged from 26 to 5565 and paternal $R P L 13 a$ transcripts that ranged from 27 to 4753 (Figure 5B). Translation of the paternal allele, as measured by red fluorescence intensities; produced protein levels that ranged from 44 to 959 a.u. and translation of the maternal allele produced blue fluorescence intensities that ranged from 15 to 399 a.u. $\left(R^{2}=0.02 ; p>0.05 ; n=30\right.$ for paternal $R P L 13 a$ and $\mathrm{R}^{2}=0.003 ; \mathrm{p}>0.05 ; \mathrm{n}=33$ for maternal RPL13a) (Figure 5B). In cells dissociated from a homozygous fly expressing PQR-RFPnols, RPL13a transcript numbers 


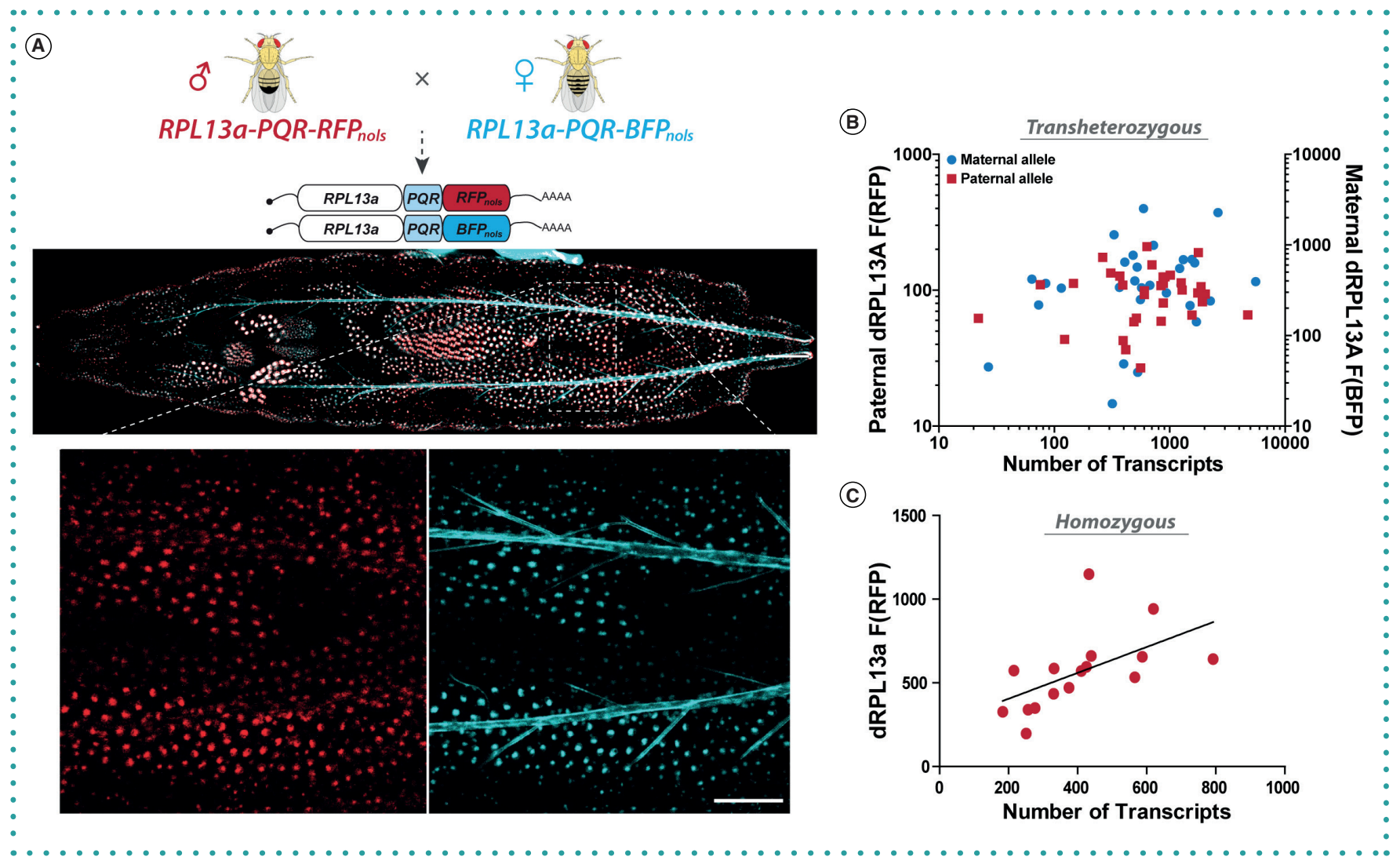

Figure 5. Tracking contribution of parental alleles at the mRNA and protein level. (A) Transheterozygous $R P L 13 a$ knock-in flies co-express $P Q R-R F P n o l s$ each time the paternal RPL13a allele is transcribed, and $P Q R-B F P n o l s$ for the maternal allele expression. Every cell that expresses $R P L 13 a$ will then produce nucleolar red and blue fluorescence. Trachea tubes are auto-fluorescent in the blue channel. (B) Examination of mRNA-protein relationships for both parental RPL13a alleles in single cells $\left(\mathrm{R}^{2}=0.02 ; \mathrm{p}>0.05 ; \mathrm{n}=30\right.$ for paternal $R P L 13 a$ and $\mathrm{R}^{2}=0.003 ; \mathrm{p}>0.05 ; \mathrm{n}=33$ for maternal $\left.R P L 13 a\right)$. (C) Drosophila $R P L 13 a$ mRNA levels can predict $R P L 13$ a protein levels with $30 \%$ accuracy in $P Q R-R F P$ nols homozygous flies $\left(R^{2}=0.3 ; p<0.05 ; n=16\right)$.

ranged from 183 to 791 transcripts, while red fluorescence intensities ranged from 196 to 1149 a.u., which resulted in a correlation of $\mathrm{R}^{2}=0.3(\mathrm{p}<0.05 ; \mathrm{n}=16)$ (Figure $5 \mathrm{C})$. Consistent with our findings in the mouse and human genomes, Drosophila $R P L 13 a$ protein levels could be predicted from absolute mRNA transcript levels with at best $30 \%$ accuracy.

The genome-wide correlation between mRNA and protein levels in cell populations is recognized to be poor, with only $40 \%$ of the variance accounted for between mRNA and protein levels [24]. This is typically attributed to regulatory mechanisms that can independently affect each step of gene expression. Measuring and correlating the levels of IgK and RPL13a mRNA and protein from single 22c10 cells shows exactly how different genes can have different mRNAprotein relationship dynamics: IgK mRNA level was a better predictor of IgK protein levels $\left(R^{2}=0.4 ; p<0.05 ; n=24\right)$, compared to $R P L 13 a$ mRNA to protein levels $\left(R^{2}=0.1\right.$; $\mathrm{p}>0.05 ; \mathrm{n}=17$ ) (Figure 4E). In addition, RPL13a mRNA was more likely to co-vary with other mRNAs, compared with RPL13a protein variation (Figures $4 \mathrm{C} \& \mathrm{E}$ ).

This approach also allows the examination of relationships between the expression of different genes and their products. Since this technique yields two metrics per gene, assaying multiple genes allows the co-correlation of mRNA and protein levels across different genes. This can be used to study the regulation and differential expression of mRNAs and proteins of multiple genes at the singlecell level. One analogous example is the use of housekeeping genes for the normalization of quantitative mRNA and protein measurements. In this case changes in $I g K$ mRNA or protein levels may be normalized to the mRNA and proteins levels of RPL13a, a commonly used housekeeping gene for normalization of expression. For example, this knock-in 22c10 cell line can be experimentally manipulated to screen for cells that exclusively upregulate the expression of $I g K$ antibodies, without affecting RPL13a. Similarly, this technique can be used to examine whether different genes are co-regulated within a single cell, under different treatment or disease conditions.

The method can be improved by faster collection and imaging of cells using automated or microfluidic chambers and using nonprecipitation approaches to isolate the total RNA. However, in its present form the protocol requires 5 days to perform CRISPR experiments and initiate clonal selection of cells carrying PQR reporters (Supplementary Figure 1A), and then $4 \mathrm{~h}$ from start of imaging to $\mathrm{qPCR}$ amplification (Supplementary Figure 2). In addition, the protocol does not require uncommon reagents or equipment and is accessible to a wide range of researchers. Because of the small quantity of starting template, certain low copy-number genes may fall below the detection range of the reverse-transcriptase or PCR polymerase 
enzymes, and the success and quality of the amplification depends on the optimization of the primer and probe conditions, and the quality of the template. With $85 \%$ efficiency primers, our results show that the average amplification failure rate is around two to three in ten cells, and is target-dependent (nine failed $R P L 13$ a reactions vs 0 failed $I g K$ reactions). Such results can be explained by variability in the RT step between $\lg K$ and $R P L 13 a \mathrm{RT}$ primers and by the substantially lower number of RPL13a transcripts (mean = 1156 transcripts) as compared with $\lg K$ (mean $=61,282$ transcripts). The failure rate may be improved with more efficient primers, and this further increases the reproducibility of the assay (Supplementary Figure 3; Troubleshooting).

Analyzing the expression of genes at both the transcriptional and translational levels from the same cell can reveal previously hidden mechanisms that produce cellular phenotypes from gene expression. In addition, insertion of $\mathrm{PQR}$ reporters into a disease-associated gene can be used to readout $\mathrm{mRNA}$ and protein responses to drugs or therapies. Finally, this protocol can easily be applied to determine how well mRNA levels of a gene of interest can serve as a proxy for protein abundance, such as in circadian controlled genes.

\section{AUTHOR CONTRIBUTIONS}

$\mathrm{IK}$ and BEC designed the experiments. IK carried out the experiments. IK and BEC analyzed the data and wrote the manuscript.

\section{ACKNOWLEDGMENTS}

The authors thank Dr Chiu-An Lo and Dr Farida Emran for assistance with experiments.

\section{FINANCIAL \& COMPETING}

\section{INTERESTS DISCLOSURE}

This work was supported by a grant from the Natural Sciences and Engineering Research Council of Canada (B.E.C.). The authors have no other relevant affiliations or financial involvement with any organization or entity with a financial interest in or financial conflict with the subject matter or materials discussed in the manuscript apart from those disclosed.

No writing assistance was utilized in the production of this manuscript.

\section{OPEN ACCESS}

This work is licensed under the AttributionNonCommercial-NoDerivatives 4.0 Unported License. To view a copy of this license, visit http://creativecommons.org/licenses/ by-nc-nd/4.0/

\section{SUPPLEMENTARY DATA}

To view the supplementary data that accompany this paper please visit the journal website at: www.future-science. com/doi/full/10.2144/btn-2018-0130

\section{REFERENCES}

Papers of special note have been highlighted as: - of interest * of considerable interest

1. Walker JM. The Protein Protocols Handbook. Humana Press, NY, USA (2009).

2. Wu M, Singh AK. Single-cell protein analysis. Curr. Opin Biotechnol. 23(1), 83-88 (2012).

3. Flatz L, Roychoudhuri R, Honda M et al. Single-cell gene-expression profiling reveals qualitatively distinct CD8 T cells elicited by different gene-based vaccines. Proc. Natl Acad. Sci. USA 108(14), 5724-5729 (2011).

4. Li GW, Xie XS. Central dogma at the single-molecule level in living cells. Nature 475(7356), 308-315 (2011)

5. Lo C-A, Kays I, Emran F, Lin T-J, Cvetkovska V, Chen Brian E. Quantification of protein levels in single living cells. Cell Rep. 13(11), 2634-2644 (2015).

- Original publication describing the protein quantitation ratioing (PQR) technique, properties, limitations, and constructs; contains detailed methodology on using PQR for protein synthesis detection.

6. Shalek AK, Satija R, Adiconis $X$ et al. Single-cell transcriptomics reveals bimodality in expression and splicing in immune cells. Nature 498(7453), 236-240 (2013).

7. Spencer SL, Gaudet S, Albeck JG, Burke JM, Sorger PK. Non-genetic origins of cell-to-cell variability in TRAIL-induced apoptosis. Nature 459(7245), 428-432 (2009).

8. Tay S, Hughey JJ, Lee TK, Lipniacki T, Quake SR, Covert MW. Single-cell NF-kappaB dynamics reveal digital activation and analogue information processing. Nature 466(7303), 267-271 (2010).

9. Darmanis S, Gallant CJ, Marinescu VD et al. Simultaneous multiplexed measurement of RNA and proteins in single cells. Cell Rep. 14(2), 380-389 (2016).

10. Albayrak C, Jordi CA, Zechner C et al. Digital quantification of proteins and mRNA in single mammalian cells. Mol Cell. 61(6), 914-924 (2016).

11. Frei AP, Bava FA, Zunder ER et al. Highly multiplexed simultaneous detection of RNAs and proteins in single cells. Nat. Methods 13(3), 269-275 (2016)

12. Pombo MA, Zheng Y, Fei Z, Martin GB, Rosli HG. Use of RNA-seq data to identify and validate RT-qPCR reference genes for studying the tomato-Pseudomonas pathosystem. Sci. Rep. 7, 44905 (2017).

13. Fujita SC, Zipursky SL, Benzer S, Ferrus A, Shotwell SL Monoclonal antibodies against the Drosophila nervous system. Proc. Natl Acad. Sci. USA 79(24), 7929-7933 (1982).

14. Curtis KM, Gomez LA, Rios C et al. EF1 alpha and RPL13a represent normalization genes suitable for RT-qPCR analysis of bone marrow derived mesenchymal stem cells. BMC Mol. Biol. 11, 61 (2010).

15. Gubern $\mathrm{C}$, Hurtado $\mathrm{O}$, Rodriguez $\mathrm{R}$ et al. Validation of housekeeping genes for quantitative real-time PCR in in vivo and in vitro models of cerebral ischaemia. $B M C \mathrm{Mol}$. Biol. 10, 57 (2009).

16. Mane VP, Heuer MA, Hillyer P, Navarro MB, Rabin RL. Systematic method for determining an ideal housekeeping gene for real-time PCR analysis. J. Biomol. Tech. 19(5), 342-347 (2008).

17. Scott MS, Boisvert FM, McDowall MD, Lamond Al, Barton GJ. Characterization and prediction of protein nucleolar localization sequences. Nucleic Acids Res. 38(21), 7388-7399 (2010).

18. Nolan T, Hands RE, Bustin SA. Quantification of mRNA using real-time RT-PCR. Nat. Protoc. 1(3), 1559-1582 (2006).

19. Li F, Vijayasankaran N, Shen AY, Kiss R, Amanullah A Cell culture processes for monoclonal antibody production. MAbs 2(5), 466-479 (2010).

20. Lo KM, Gillies SD. High level expression of human proteins in murine hybridoma cells: induction by methotrexate in the absence of gene amplification. Biochim Biophys. Acta 1088(2), 217-224 (1991).

21. Zhou X, Liao WJ, Liao JM, Liao P, Lu H. Ribosomal proteins: functions beyond the ribosome. J. Mol. Cell Biol. 7(2), 92-104 (2015).

22. Greenbaum D, Colangelo C, Williams K, Gerstein M. Comparing protein abundance and mRNA expression levels on a genomic scale. Genome Biol. 4(9), 117 (2003).

23. Maier T, Guell M, Serrano L. Correlation of mRNA and protein in complex biological samples. FEBS Lett. 583(24), 3966-3973 (2009).

24. Schwanhausser B, Busse D, Li N et al. Global quantification of mammalian gene expression control. Nature 473(7347), 337-342 (2011). 